\title{
The healing of damage after the plastic deformation of metals
}

\author{
S.V. Smirnov \\ Institute of Engineering Science, Ural Branch of Russian Academy of Sciences \\ 34 Komsomolskaya st., Ekaterinburg, 620219, Russia. \\ svs@imach.uran.ru
}

\begin{abstract}
The general regularities of damage healing during the annealing after cold deformation of metal materials are presented in this paper. In categories of damage mechanics the kinetic equations of damage healing during recovery and recrystallization are formulated. Diagrams of damage healing for some metal alloys are presented. The example of use of investigation results for optimization of industrial technology of pipes drawing is presented.
\end{abstract}

KEYwORDS. Deformation damage; Metal forming; Fracture; Healing of damage; Prediction of fracture.

\section{INTRODUCTION}

A ccording to the current conception of metal physics, the fracture of metal materials is not a one-act catastrophic phenomenon, but a regular process of appearance and development of defects, which is in mechanics referred to as damage accumulation (plastic loosening, damageability, cracking, etc.). Pure brittle damage is possible only in metals with a large covalent component in the interatomic bond. The form and shape of defects, as well as the velocity of their propagation, depend on metal behaviour and thermomechanical loading conditions; however, the active role of plastic deformation is invariant here, and it reveals itself on the macro or micro scale.

So the technological cold plastic deformation of metal (rolling die-forging, etc.) from the first stages is accompanied by microscopic defects of continuity. The development of damage with the accumulation of deformation can result in the appearance of macroscopic defects or even the division of the body under deformation into separate parts, i.e. in defective products: this is definitely inadmissible. Macroscopic defects can be revealed easily (external by visual observation, internal by an introscopy method), but correction is either impossible or requires that the defective bulk of the metal should be removed. One of the methods for avoiding macro-damage is multi-stage deformation, with intermediate annealing at the end of every stage, which provides metal softening and, above all, the restoration of metal plasticity (i.e. the ability of the metal to be deformed without fracture). The amount of deformation in a separate stage is established intuitively, proceeding from one's practical experience. To understand the technology of manufacturing cold-deformed products with annealing, it is necessary to give a mathematical description (within the above-mentioned model) of how the restoration of the reduction of micro-damage proceeds under annealing.

As distinct from macro-defects, micro-discontinuities are harder to detect under service conditions. Industry lacks the means of checking micro-flaws, so, therefore all metal products have micro-flaws which can affect the efficiency of machine parts. It has been ascertained hat they influence fatigue life [1]. Therefore it is important to study the mechanisms of eliminating (or healing) micro-flaws, i.e. the mechanisms for the restoration of the margin of metal plasticity by heat treatment and the ways of making it more efficient. This is the subject matter of the present paper. 


\section{DAMAGE MODEL}

7 he deformational criteria of damage and the phenomenological theories based on a certain hypothesis of damage accumulation are widespread in mechanics [1-8]. In the description of damage under developed plastic conditions, the deformational approach is also popular (see, for example, the survey found in [9]). Historically, the problem of damage in plastic deformation was initially considered in terms of technological interests on the basis of empiric criteria and fracture models. This approach allowed some simple applied problems to be solved, but hampered the study of the general rules of metal damage under the complex stress-strain state. In mechanics, the progress in the development of the notion of metal damage under plastic deformation is connected with the appearance of kinetic theories of dispersed fracture (damage mechanics) [1, 4-12].

The process of damage under plastic deformation can be represented in a different way in terms of the damage mechanics as

$$
\frac{d \omega}{d \Lambda}=f\left(\omega, s_{1}, s_{2}, \ldots \ldots\right)
$$

where $\omega$ is a characteristic of metal damage; $S_{1}$ and $S_{2}$ are thermomechanical loading parameters depending on the loading conditions. Before loading $\omega=0$ while $\omega=1$ when the fracture happens. Intermediate values of $\omega$ characterize a level of development of micro-defects.

The kinetic equation Eq.1 was first proposed by L.M. Kachanov $[2,3]$ to describe damage in creep, and was later used by a number of authors to describe damage under plastic deformation. The most well-known model of metal damage under plastic deformation to be used for making practical calculations is the linear model authored by V. L. Kolmogorov $[1,4,7]$

$$
\frac{d \omega}{d \Lambda}=\frac{1}{\Lambda_{f}}
$$

where $\Lambda_{f}$ is metal plasticity defined as limiting (at the instant of fracture) accumulated amount of shear strain $\Lambda$ in deformation under constant stress state characterized by the stress state index $k_{1}$ and the Lode-Nadai parameter $k_{2}$ :

$$
k_{1}=\frac{\sigma \sqrt{3}}{\sigma_{s}} ; k_{2}=\frac{2 \sigma_{2}-\sigma_{1}-\sigma_{3}}{\sigma_{1}-\sigma_{3}}
$$

where $\sigma=\left(\sigma_{1}+\sigma_{2}+\sigma_{3}\right) / 3$ is mean normal stress; $\sigma_{1,} \sigma_{2}, \sigma_{3}$ are main normal stresses; $\sigma_{s}$ is equivalent stress.

Note that in the literature there is no consensus on the form of the kinetic equation, and it is generally chosen by authors on the basis of hypothetical ideas or published fragments of metal-physic research data. Therefore in this paper we will use an adaptive model of damage accumulation [12, 15]. This model has been formulated from the analysis of experimental data on changes in metal density under plastic deformation and heat treatment after plastic deformation.

A general adaptive model of damage was formulated to describe damage accumulation under conditions of the experimental stepwise change in the stress-strain state, at a later date model was developed in some others forms. When the stress state changes, the rate of damage variation on the adaptation portion is evaluated as follows:

$$
\frac{d \omega}{d \lambda}=\left[1+c_{1}\left(1-e^{-c_{2} \Delta k_{1 i}}\right)\left(1-\frac{\lambda}{\lambda_{a}}\right)^{c_{3}}\right] \frac{1}{\Lambda_{f}}
$$

where $\Delta k_{1 i}$ is the increment of the stress state index at $i$-stepwise of loading; $\lambda=0 \ldots \lambda_{a}$ is a current amount of shear strain on the adaptation portion; $\lambda_{a}$ is the length of the adaptation portion; $c_{1}, c_{2}$ and $c_{3}$ are empiric factors.

When the direction of deformation changes, the rate of damage accumulation decreases, and on the adaptation portion it can be determined by the formula

$$
\frac{d \omega}{d \lambda}=\left\{1-c_{4}\left|\vartheta_{i}\right|\left(1-\omega_{i-1}\right)^{c_{5}} e^{-c_{6}(i-1)}\right\} \frac{1}{\Lambda_{f i}}
$$

where $\vartheta$ is the angle characterizing the change in the loading path in Ilyushin's phase space of deformations, which can be taken as a parameter for the quantitative evaluation of deformation non-monotonicity; $\omega_{i-1}$ is the damage on the portion 
preceding the $i$-th change in the direction of deformation; $c_{5}, c_{6}$ and $c_{7}$ are empirical coefficients. The linear model and adaptive model yield identical results at the simple monotonic deformation.

\section{INVESTIGATION TECHNIQUE}

o investigate the general regularities of damage healing during the annealing after cold deformation was the purpose of this paper. Notice that the restoration of the margin of plasticity in metals alter cold deformation has been studied for a number of years, the results being published in [5, 7, 13, 14]. The following technique [13, 14] has been developed for solving this problem (Fig. 1).

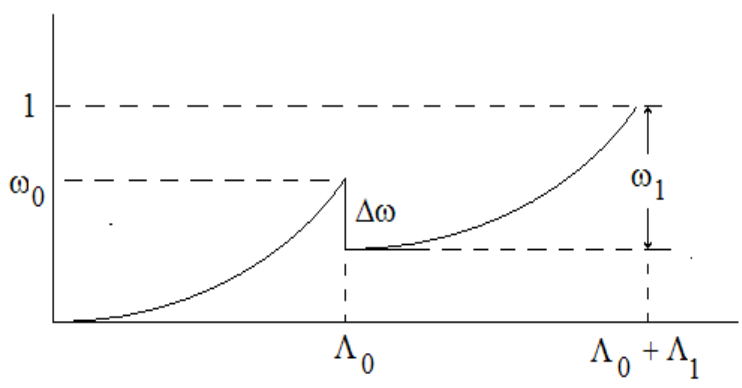

Figure 1: To definition of the damage healing under annealing after deformation

Tests are performed on metal, with plasticity $\Lambda_{f}=\Lambda_{f}\left(k_{1}, k_{2}\right)$ already known. Test specimens undergo different amounts of plastic strain $\Lambda_{0}$, each specimen being deformed to different amounts of damage $\omega_{0}$. Therefore, all the specimens undergo annealing in accordance with the chosen regime ( $T$ is temperature and $t$ is annealing duration). Damage decrease by the value $\Delta \omega$ takes place in annealing. After annealing once again, all the specimens undergo plastic deformation in the same direction up to fracture. Value of $\Delta \omega$ for each specimen can be calculated from a facture criterion

$$
\omega_{0}-\Delta \omega+\omega_{1}=1
$$

where:

$$
\omega_{0}=\int_{0}^{\Lambda_{0}} \frac{d \Lambda}{\Lambda_{f}(k)} ; \omega_{1}=\int_{\Lambda_{0}}^{\Lambda_{0}+\Lambda_{1}} \frac{d \Lambda}{\Lambda_{p}(k)}
$$

$\Lambda_{0}=2 \sqrt{3} \ln \left(d_{0} / d_{1}\right) ; \Lambda_{2}=2 \sqrt{3} \ln \left(d_{1} / d_{f}\right) ; d_{0}, d_{1}$ and $d_{f}$ are the initial diameter, diameter before annealing and diameter after fracture of specimens.

The stress state index for cylindrical specimens is calculated as $[4,8]$

$$
k=\left(1+\frac{3}{4} \frac{d}{R}(\Lambda)\right)
$$

where $\frac{d}{R}(\Lambda)$ is a Bridgman's parameter which characterizes a neck form of specimen.

\section{RESULTS AND DISCUSSION}

A $\mathrm{n}$ example of the damage-time history for low-carbon $0.2 \% \mathrm{C}$ steel at a temperature of $600^{\circ} \mathrm{C}$ is shown in Fig 2 . The lower curve $\omega=0$ illustrated that the annealing of the blank to be deformed, for example, of hot-rolled metal, can lead to higher plasticity due to the healing of the micro-damage that appears in the hot rolling stage. The curves have three distinctive parts: $\mathrm{AB}$ is a rapid exponential decrease of damage due to a recovery processes; $\mathrm{BC}$ is a considerable deceleration (and even stopping) of healing due to incubation period of the recrystallization; and CD is further acceleration of the process due to a recrystallization. 


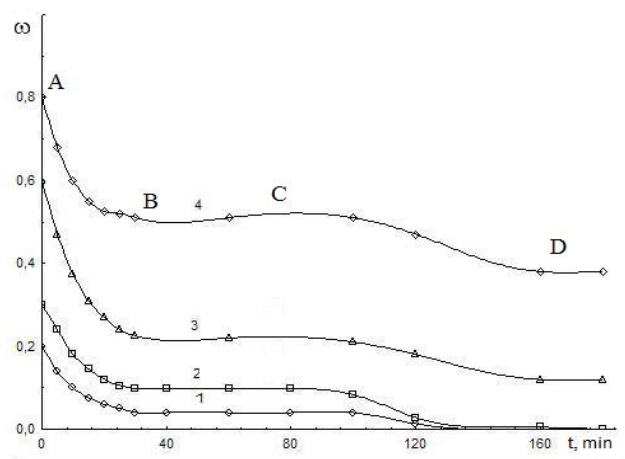

Figure 2: Damage healing during annealing (at 6000C) of carbon steel $0.2 \% \mathrm{C}$.

A decrease of damage due to a recovery processes can be described by the equation

$$
\omega=\omega_{0} \exp \left[\frac{t}{t_{r}^{2}}\left(2 t_{r v}-t\right) \ln \frac{\omega_{r v}}{\omega_{0}}\right]
$$

where $t_{r v}$ is recovery period, $\omega_{r v}$ is the bottom limit level of healing of the damage due to the recovery.
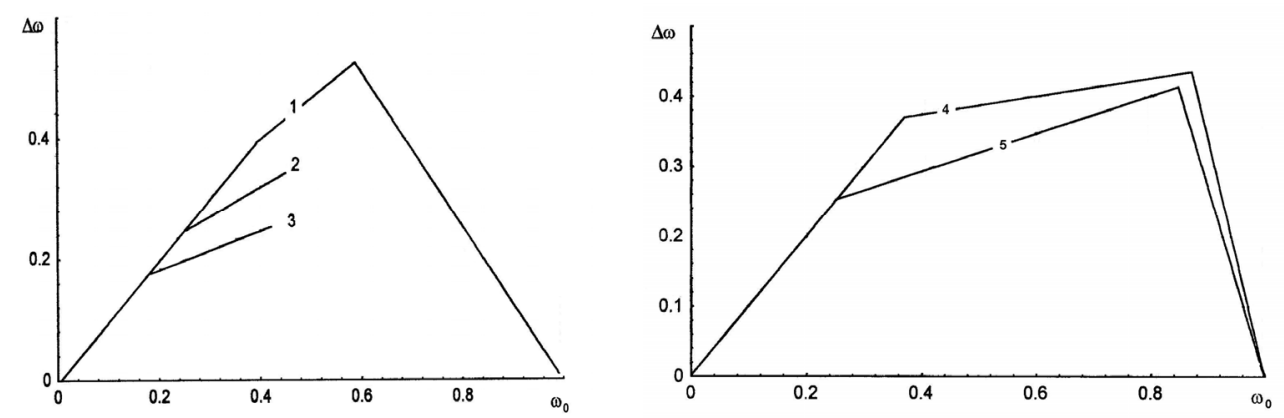

Figure 3: Diagrams of damage healing of some metal alloys: titanium alloys containing different amount of $A l$ and $M n(1-0.8 \% A l+$ $0.8 \% M n ; 2-1.5 \% A l+1 \% M n ; 3-3.5 \% A l+1.5 \% M n) ; 4$ - low-alloyed $C r ; 5-C r+35 \% F e$.

A decrease of damage due to a recrystallization processes can be described by the equation

$$
\omega=\left(\omega_{r v}-\omega_{r c}\right) \exp \left[-B\left(\frac{t-t_{r c}^{0}}{t_{r c}-t_{r c}^{0}}\right)^{k}\right]+\omega_{r c}
$$

where $t_{r c}$ is duration of the incubation period, $\omega_{r c}$ is the bottom limit level of healing of the damage due to the recrystallization.

Fig. 3 shows diagrams illustrating the degree of damage healing for some alloys in coordinates $\left(\omega_{0}, \Delta \omega\right)$. On value of the amount of $\Delta \omega$ one can estimate the completeness of the healing of micro-defects appearing in the pre-load stage. (Bear in mind, if $\Delta \omega=\omega_{0}$ the healing is complete, whereas if all the micro defects remain in the metal). Healability is different for different alloys. Recrystallization annealing leads to the complete healing of deformation damage if it is less than some value $\omega^{*}$. When $\omega^{*}<\omega_{0}<\omega^{* *}$ there is partial damage healing, and a certain part of deformational defects remains in the metal. The researches executed by SEM technique, showed that at this stage defects are micro pores (Fig. 4a). When $\omega_{0}<$ $\omega^{* *}$ the residual damage increases more intensively, micro pores coalesce, a pores and micro cracks is formed (Fig.4b). Investigations have shown that recrystallization annealing results in the healing of micro-discontinuities of sub-grain size (i.e. under $2-5 \mu \mathrm{m}$ ) by intensive surface diffusion of vacancies when they are crossed by the moving inter- grain boundary of the grain being recrystallized. Values of $\omega^{*}$ and $\omega^{* *}$ is different for different alloys but are usually are in ranges $\omega^{*}=$ $0.2-0.5$ and $\omega^{* *}=0.6-0.8$. 


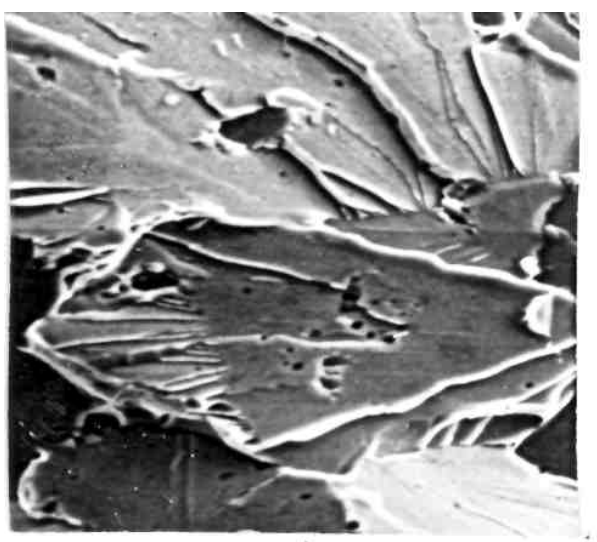

(a)

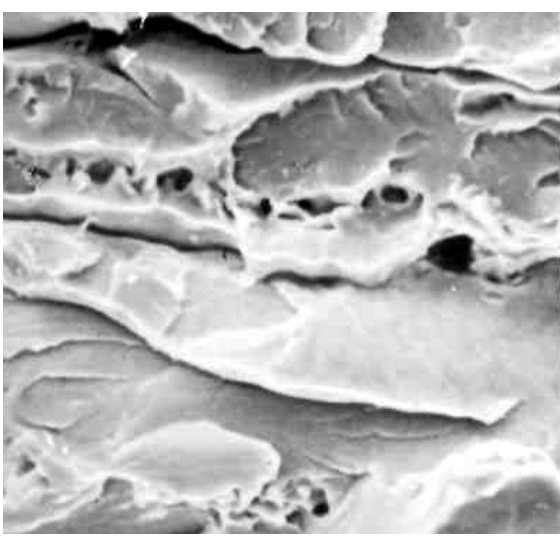

(b)

Figure 4: Micro pores and crack which was formed of coalescence of micro pores (are shown by arrows). Material is deformed carbon steel $0.1 \% \mathrm{C}$ after annealing (at $700^{\circ} \mathrm{C}, 1$ hour). Values of the initial damage: $a-\omega_{0}=0.32 ; b-\omega_{0}=0.7$ (magnification x1000).

Calculation and analysis of damage accumulated in metal allow to optimize technology process of plastic treatment. One of number of practical examples may be given [16]. At the Pervouralsk Pipe-Making Plant (Russia) pipes of carbon steel $0.45 \% \mathrm{C}$ for poles has been produced by cold rolling. Existing equipment did not allow to satisfy the demand for type product. To increase a volume of production it was offered to be produce at automated triple drawing line. One of major questions stated for engineers was a question of damage of pipes during drawing because the manufacturing line design did not suppose the intermediary annealing. Theoretical calculations allowed to choose an optimal drawing parameters, when the level of residual damage was not dangerous (Fig.5). The experimental investigations of the relative changes of density $\Delta \rho / \rho_{o}$ and then industrial tests of theoretical results show a validity of prediction.

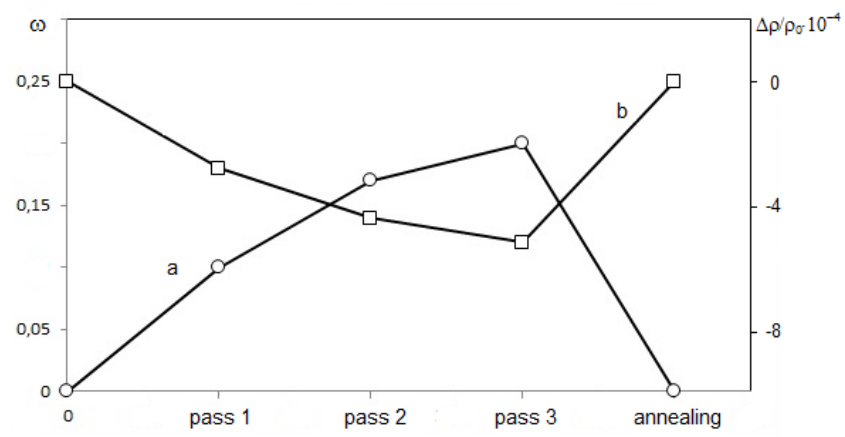

Figure 5: Changes of damage $\omega$ (a) and density $\Delta \rho / \rho_{0}$ (b) under drawing and annealing of $0.45 \% \mathrm{C}$ carbon steel pipes.

\section{CONCLUSIONS}

I $\mathrm{n}$ this work the equations of damage healing during recovery and recrystallization are in categories of damage mechanics are formulated. Diagrams of damage healing for some metal alloys are defined. It is shown that recrystallization annealing leads to the complete healing of deformation damage $\omega$ if it is less than some value $\omega^{*}$. When $\omega^{*}<\omega_{0}<\omega^{* *}$ there is partial damage healing, and a certain part of deformational defects remains in the metal. The example of use of investigation results for optimization of industrial technology of pipes drawing is presented.

\section{ACKNOWLEDGEMENTS}

This work has been executed according to plan of the project number 12-T-1-1010 of the UB RAS Research Program and was supported by a grant from the Russian Foundation for Basic Research, contract number 11-08-12083. 
The author expresses gratitude to the professor V.L. Kolmogorov for initiation of this research and discussion of its results.

\section{REFERENCES}

[1] V.G. Burdukovsky, V.L. Kolmogorov, B.A. Migachev, J. Mater. Process. Technol., 55 (1995) 292.

[2] L.M. Kachanov, Docladi Academii Nauk SSSR, Otdelenie Tekhnicheskih Nauk 8 (1958) 67 - 65 (in Russian).

[3] L.Kachanov, Introduction to Continium Damage Mechanics, Martinus Nijhoff Publishers, Dordrecht, (1986) 135.

[4] V.L. Kolmogorov, Metallurgiya (1970) 232 (in Russian).

[5] A.A. Bogatov, O.I. Mizhiritsky, S.V. Smirnov, Metallurgiya (1984) 144 (in Russian).

[6] J. Lemaitre, A Course on Damage Mechanics, Springer, Berlin (1987).

[7] V.L. Kolmogorov, In: Materials Processing Defects, S.K. Gosh, M. Predeleanu (Eds.), Elsevier, Amsterdam (1995) 87

[8] V.L. Kolmogorov, Wear 194 (1996) 71.

[9] A.G. Atkins, In: An Anniversary Volume in Honour of George R. Irvin's 90th Birthday, H.P. Rossmanith. A.A. Balkema (Eds.), Brookfield, Rotterdam (1997) 327.

[10] M. Oyane, Bulletin of JSME, 15, 90 (1972) 37.

[11] Z.J. Luo, W.H. Ji, N.C. Guo et alii, Journal of Material Processing Technology 30 (1992) 31.

[12] S.V. Smirnov, Key Engineering Materials, 528 (2013) 61.

[13]V.L. Kolmogorov, S.V. Smirnov, Journal of the Materials Processing Technology, 74 (1998) 83.

[14] A.A. Bogatov, V.L. Kolmogorov, S.V. Smirnov, Izvestiya VUZov, Chernaya Metallurgiya, 12 (1978) 43 (in Russian)

[15] S.V. Smirnov, T.V. Domilovskaya, A. A. Bogatov, In: Materials Processing Defects, S.K. Gosh, M. Predeleanu (Eds.), Elsevier, Amsterdam, (1997) 71. 